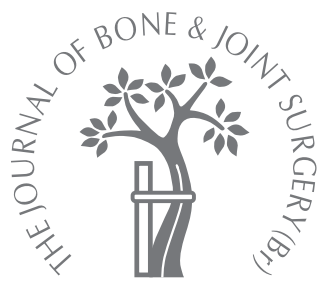

R. Wedin, H. C. F. Bauer

From Karolinska University Hospital, Stockholm, Sweden

\title{
Surgical treatment of skeletal metastatic lesions of the proximal femur
}

\author{
ENDOPROSTHESIS OR RECONSTRUCTION NAIL?
}

\begin{abstract}
We report positive and negative factors associated with the most commonly-used methods of reconstruction after pathological fracture of the proximal femur. The study was based on 142 patients treated surgically for 146 metastatic lesions between 1996 and 2003. The local rate of failure was $10.3 \%$ (15 of 146). Of 37 operations involving osteosynthetic devices, six failed (16.2\%) compared with nine $(8.3 \%)$ in 109 operations involving endoprostheses. Of nine cases of prosthetic failure, four were due to periprosthetic fractures and three to recurrent dislocation. In the osteosynthesis group, three (13.6\%) of 22 reconstruction nails failed. The two-year risk of re-operation after any type of osteosynthesis was 0.35 compared with 0.18 after any type of endoprosthetic reconstruction $(p=0.07$ ). Endoprosthetic reconstructions are preferable to the use of reconstruction nails and other osteosynthetic devices for the treatment of metastatic lesions in the proximal third of the femur.
\end{abstract}

The aim of treatment of a patient with metastatic bone disease and a pathological fracture is to provide stable and secure fixation which allows immediate weight-bearing and restoration of function and which will outlast the patient. A common location for failures of fixation of pathological fractures is the proximal femur. The best method of reconstruction is uncertain. Some surgeons prefer endoprosthetic replacement while others advocate the use of reconstruction nails or other osteosynthetic devices with or without the adjunct of cement. We report a retrospective review of a large series of patients to identify the positive and negative factors related to each method.

\section{Patients and Methods}

Our retrospective study was based on a consecutive series of 159 patients who had surgical treatment for metastatic lesions of the proximal femur at the Karolinska Hospital between 1996 and 2003. Sixteen patients referred after failure of their primary surgery at other hospitals were excluded. Since the aim of the study was to assess the reasons for reconstructive failures, one patient who had undergone a Girdlestone procedure was also excluded, leaving 142 patients in the study. Four had bilateral fractures giving a total of 146 operations for analysis.

There were 73 women and 69 men with a median age of 69 years (33 to 91). The breast was the most common site of the primary tumour $(32 \%)$, followed by the prostate $(25 \%)$ and the lung $(11 \%)$. The median elapsed time from the date of diagnosis of cancer until operation for the fracture was 2.5 years (0 to 18). The median time varied for the different primary lesions: for the lung it was six months, for the kidney one year, for the prostate two years and for the breast six years. Only $16 \%$ of the patients had a solitary skeletal metastasis; $54 \%$ had numerous skeletal metastases and $30 \%$ also had non-skeletal metastases.

Metastases in the femoral head and neck predominated (55 of 146, 38\%), followed by subtrochanteric $(51,35 \%)$ and intertrochanteric lesions $(40,27 \%)$. A complete fracture was evident in 126 (86\%) of the metastases whereas $20(14 \%)$ were operated on for impending fractures. During the eight years of the study there was no strict definition of impending fracture. Generally, prophylactic stabilisation was performed in the presence of considerable cortical destruction associated with pain on weight-bearing or failure to improve after radiotherapy. All 37 prostatic and eight renal lesions were associated with a complete fracture as opposed to 38 of 48 lesions of the breast $(79 \%)$.

Operative treatment. We used a range of implants and osteosynthetic material. The unreamed femoral nail (Synthes Stratec Medical, Oberdorf, Switzerland) was the locked intramedullary device. In seven cases a spiral blade inserted into the femoral head was added so 
Table I. Types of reconstruction

\begin{tabular}{lrrrr}
\hline & $\begin{array}{l}\text { Femoral head } \\
\text { and neck }\end{array}$ & Intertrochanteric & Subtrochanteric & Total \\
\hline Hemiarthroplasty & 31 & 12 & 8 & 51 \\
Bipolar prosthesis & 1 & 2 & 1 & 4 \\
Regular THR & 19 & 12 & 14 & 45 \\
Tumour prosthesis & 2 & 2 & 1 & 5 \\
Reconstruction prosthesis & 1 & 0 & 3 & 4 \\
Reconstruction nail & 0 & 4 & 18 & 22 \\
DHS & 0 & 8 & 4 & 12 \\
Locked intramedullary nail & 0 & 0 & 2 & 2 \\
Hip screws & 1 & 0 & 0 & 1 \\
Total & 55 & 40 & 51 & 146 \\
\hline * THR, total knee replacement & & &
\end{tabular}

that it was classified as a reconstruction nail. The other reconstruction nail used was the proximal femoral nail (Synthes Stratec Medical). The Dynamic Hip Screw (DHS) (Synthes Stratec Medical) was the only sliding screw plate used. The Austin Moore hemiarthroplasty (Corin Medical, Cirencester, UK) was the most frequently used implant, followed by the Charnley (DePuy, Leeds, UK) and Spectron (Smith \& Nephew, Memphis, Tennessee) total hip replacements (THRs).

Operative treatment was divided into en bloc resection and reconstruction of the involved bone using a tumour prosthesis (five operations), intra-lesional curettage (115) or stabilisation (26). Resection surgery was mainly used in patients with a good prognosis. Reconstruction was achieved by an endoprosthesis in 109 operations and by osteosynthesis in 37. A hemiarthroplasty was the most common method of endoprosthetic reconstruction (51 operations) followed by conventional THR (45), a tumour prosthesis (five), a reconstruction prosthesis (four) and a bipolar prosthesis (four). One of the hemiarthroplasties and two of the reconstruction prostheses were used without cement. Of the 37 cases of osteosynthesis, local excision of the tumour by curettage and cementation was performed in nine operations; in six of 22 reconstruction nail procedures and in three of 12 DHS operations. Prostheses were used in 54 of 55 reconstructions of the femoral head and neck. One patient had hip screws because the fracture was not suspected of being pathological at the time of operation. In 40 intertrochanteric fractures, 28 prostheses, eight DHS and four reconstruction nails were used. In 51 subtrochanteric fractures, reconstruction was achieved with 27 prostheses, 18 reconstruction nails, four DHS and two intramedullary nails (Table I).

Re-operations. Failure of treatment requiring re-operation at the same site was considered to be the end-point of the study. No patient was lost to follow-up. The reasons for reoperation were subdivided into four major causes: periprosthetic fracture, nonunion, technical error and miscellaneous.

Survival analysis. This was performed using Kaplan-Meier analysis.

\section{Results}

Ten patients were still alive at the last follow-up in February 2005 at a median of 1.6 years after operation.

Complications. The overall rate of systemic complications was $4.1 \%$ (six operations) and that of local complications $3.4 \%$ (five operations). All 11 complications occurred among the 109 prosthetic replacements and none in the 37 osteosyntheses. Two patients with pulmonary emboli, one each with a perforated colon and cardiac failure, made uneventful recoveries. One patient died from a cerebrovascular accident one day after surgery and another from multiple organ failure at four days. The local complications included four infections and one nerve injury. In the prosthetic replacement group the systemic and local complication rates, excluding prosthetic dislocations, were $5.5 \%$ and $4.6 \%$, respectively. Fifteen $(13.8 \%)$ patients had at least one dislocation; three of whom required revision for recurrent dislocation. The rate of dislocation for hemiarthroplasty was 4\% (2 of 51) and for THR 22.2\% (10 of 45). Three of the four bipolar prostheses dislocated. In the group of dislocated THRs, eight of $32(25 \%)$ were Charnley THRs (head size $22 \mathrm{~mm}$ ) used until July 2002 and two of $13(15.4 \%)$ with the Spectron system (head size $28 \mathrm{~mm}$ ) which was used thereafter. There was no prosthetic dislocation in the nine patients treated with a reconstruction or tumour prosthesis. There was no obvious difference in the complications occurring in patients with a complete or an impending fracture.

Frequency of failures. Fifteen of the $146(10.3 \%)$ procedures failed necessitating re-operation. The median time to reoperation was seven months (Table II). Of the 109 endoprostheses, nine required re-operation $(8.3 \%)$, as did four $(8 \%)$ of the 51 hemiprostheses and five of the 45 THRs (11.1\%). All but three prostheses were cemented.

Of 37 operations involving fracture fixation six were failures $(16.2 \%)$. Three of the 22 reconstruction nails $(13.6 \%)$ required re-operation as did three of the 12 sliding hip screws $(25 \%)$. In nine patients treated by local excision of the tumour and cement, there was one failure $(11.1 \%)$ compared with five in the group of 28 patients $(17.9 \%)$ operated by stabilisation only. 
Table II. Details of the re-operations performed

\begin{tabular}{|c|c|c|c|c|c|c|c|c|}
\hline Case & Gender* & $\begin{array}{l}\text { Age } \\
\text { (yrs) }\end{array}$ & $\begin{array}{l}\text { Primary } \\
\text { tumour }\end{array}$ & Localisation & Primary operation $^{\dagger}$ & $\begin{array}{l}\text { Time }^{\ddagger} \\
\text { (mths) }\end{array}$ & $\begin{array}{l}\text { Reason for } \\
\text { re-operation }\end{array}$ & $\begin{array}{l}\text { Reconstruction } \\
\text { at re-operation }\end{array}$ \\
\hline 1 & $\mathrm{~F}$ & 49 & Breast & Cervical & THR & 9 & $\begin{array}{l}\text { Periprosthetic } \\
\text { pathological fracture }\end{array}$ & THR, long stem \\
\hline 2 & $\mathrm{~F}$ & 57 & Sarcoma & Cervical & THR & 26 & $\begin{array}{l}\text { Periprosthetic } \\
\text { pathological fracture }\end{array}$ & THR, long stem \\
\hline 3 & $\mathrm{~F}$ & 72 & Breast & Trochanteric & THR & 24 & $\begin{array}{l}\text { Periprosthetic } \\
\text { pathological fracture }\end{array}$ & $\begin{array}{l}\text { Plate/plate/tumour } \\
\text { prosthesis }\end{array}$ \\
\hline 4 & $\mathrm{~F}$ & 72 & Breast & Trochanteric & THR & 1 & Technical error & $\begin{array}{l}\text { Revision of femo- } \\
\text { ral component }\end{array}$ \\
\hline 5 & $M$ & 75 & Myeloma & Cervical & THR & 1 & Technical error & Cup revision \\
\hline 6 & $\mathrm{~F}$ & 37 & Breast & Trochanteric & Cemented hemiarthroplasty & 36 & Technical error & $\begin{array}{l}\text { THR + acetabular } \\
\text { ring }\end{array}$ \\
\hline 7 & $\mathrm{~F}$ & 51 & Breast & Subtrochanteric & Cemented hemiarthroplasty & 6 & $\begin{array}{l}\text { Periprosthetic } \\
\text { pathological fracture }\end{array}$ & THR, long stem \\
\hline 8 & $\mathrm{~F}$ & 67 & Lung & Cervical & Cemented hemiarthroplasty & 1 & $\begin{array}{l}\text { Suspected loosening/ } \\
\text { pain }\end{array}$ & THR, long stem \\
\hline 9 & $\mathrm{M}$ & 77 & Myeloma & Subtrochanteric & Cemented hemiarthroplasty & 1 & Technical error & THR, long stem \\
\hline 10 & $\mathrm{~F}$ & 39 & Breast & Subtrochanteric & Reconstruction nail & 11 & Nonunion & THR, long stem \\
\hline 11 & $M$ & 52 & Kidney & Subtrochanteric & Reconstruction nail & 27 & Nonunion & Tumour prosthesis \\
\hline 12 & $\mathrm{~F}$ & 76 & Breast & Trochanteric & Reconstruction nail & 1 & Technical error & Reconstruction nail \\
\hline 13 & $\mathrm{~F}$ & 59 & Breast & Trochanteric & DHS & 7 & Nonunion & THR, long stem \\
\hline 14 & M & 75 & Prostate & Trochanteric & DHS & 1 & Stress fracture & THR, long stem \\
\hline 15 & $\mathrm{~F}$ & 80 & Breast & Subtrochanteric & DHS & 19 & $\begin{array}{l}\text { Osteoporosis of } \\
\text { femoral head }\end{array}$ & THR \\
\hline
\end{tabular}

* $\mathrm{F}$, female; $\mathrm{M}$, male

† THR, total hip replacement; DHS, dynamic hip screw

$\ddagger$ time to re-operation

Reasons for re-operation. Of the 15 re-operations, five were attributable to technical errors, four to periprosthetic pathological fractures, three to nonunion, and three to miscellaneous reasons (Table II). Of the five technical errors, one was because of a misplaced reconstruction nail, one to a loose piece of cement entering the joint space leading to pain and three were associated with repeated dislocations. Three of the four periprosthetic fractures occurred immediately distal to the femoral component between nine and 24 months after the primary surgery. The fractures were considered to be pathological since histological evidence of growth of the tumour was present in all three. In the fourth case of periprosthetic fracture, the fracture developed along the stem of a cemented hemiprosthesis leading to pain. No evidence of growth of the tumour was found in the biopsy specimen collected at the re-operation six months after primary surgery.

The three cases of nonunion occurred in two patients with reconstruction nails, and one with a DHS which broke at the site of the fracture, between seven and 27 months after the primary surgery. There were no radiological, surgical or histological findings of tumour at the site at reoperation.

Of the miscellaneous reasons for re-operation, one was avascular necrosis of the femoral head 19 months after a subtrochanteric fracture had been treated successfully by a DHS. A second case was caused by a fall two weeks after primary surgery leading to breakage of a DHS. The third was in a patient who had severe load-related pain after reconstruction of a fracture of the femoral neck with a cemented hemiprosthesis. The radiological appearances suggested inadequate fixation of the prosthesis, but this was not confirmed at re-operation two weeks after the primary surgery.

Treatment of failures. Procedures performed for the 15 failures involved 13 endoprosthetic replacements, one reconstruction nail and one femoral plate. The patient who received the femoral plate suffered two more failures of reconstruction requiring two subsequent surgical procedures (Table II).

Patient and tumour features. Breast cancer was the primary tumour with the highest rate of re-operation, nine of 15 failures (Table II). Re-operations were more common in the intertrochanteric (six of 15) than in the subtrochanteric (five of 15) or the cervical (four of 15) region.

Survival. The Kaplan-Meier survival rate for the whole series of 142 patients was 0.3 at one year after surgery, 0.1 at two years and 0.07 at three years.

The median survival after operation for a pathological fracture for patients requiring re-operation was 15 months compared with five for patients not requiring further surgery $(p=0.03)$. Of the ten patients who are still alive, one has had further surgery. Kaplan-Meier estimates showed that the risk of re-operation increased linearly with times, being 0.14 at one year and 0.20 at two years. Only 14 patients were still at risk of re-operation at two years.

Univariate analysis of failures. The two-year risk of re-operation after any type of osteosynthesis was 0.35 compared with 0.18 after any type of endoprosthetic reconstruction $(\mathrm{p}=0.07)$.

\section{Discussion}

In 1999 we reported a study of 192 patients treated surgically for 228 pathological fractures of the long bones 
between 1986 and $1995 .^{1}$ The most important risk factor for failure of treatment was the length of survival after operation. In addition, reconstruction based on prosthetic as opposed to osteosynthetic devices appeared to be safer. Consequently, we have favoured endoprosthetic replacements rather than osteosynthesis for pathological fractures. We have now evaluated our results for proximal femoral lesions from 1996 to 2003. One aim was to determine whether, with experience, our results were better. Disappointingly, they were not. The overall rate of failure from 1986 to 1995 for the proximal femur was $9 \%$ (ten of 108) as compared with $10.3 \%$ (15 of 146) in the present study. By performing fewer osteosyntheses, failure due to local progression of disease, poor initial fixation, nonunion and stress fracture has decreased, but we encountered technical errors in endoprosthetic surgery and periprosthetic fractures. Nevertheless, osteosynthetic devices were still responsible for six of 15 failures and were associated with a higher rate of failure than for prostheses.

In the present study, the rate of dislocation was $13.8 \%$ and three prostheses were revised because of recurrent dislocation. The rate was more than five times greater in THRs $(22.2 \%)$ than hemiarthroplasties $(4 \%)$. A dislocation rate of $22.2 \%$ for pathological fractures treated by THR is high, but is comparable with that in published data for nonpathological displaced femoral fractures.,3 Of four patients treated surgically with bipolar prostheses, three had a dislocation. This finding makes us hesitant to use bipolar prostheses in pathological fractures.

Four of 109 (3.7\%) endoprosthetic replacements led to a periprosthetic fracture which is comparable with the incidence of periprosthetic non-pathological fractures after revision $(2.4 \%) .{ }^{4}$ If a reconstruction nail or long-stemmed prosthesis had been chosen, at least three of the four fractures could probably have been avoided. Some authors propose that long-stemmed prostheses should be used routinely while others oppose this view because of the increased risk of cardiac arrest. ${ }^{5-7}$ The use of long-stemmed femoral components routinely when the incidence of periprosthetic fracture is only $4 \%$ is questionable. Additionally, in the three cases in which the fracture was at the tip of the femoral component, biopsy showed tumour cells. The aetiology of these fractures may be intra-operative tumour-cell seeding since the fractures occurred at a mean of 20 months after operation. The use of long-stemmed prostheses would not have prevented this.

Six of $37(16.2 \%)$ osteosynthetic reconstructions failed and warranted re-operation. When local removal of the tumour was followed by cementation, one of nine $(11 \%)$ reconstructions failed as compared with five failures in the group of 27 patients (19\%) in whom curettage alone was used. Many previous studies support the addition of cement in osteosynthesis of pathological fractures. ${ }^{8-10}$

The overall rate of re-operation was $8.3 \%$ (nine of 109) in the prosthetic and $16.2 \%$ (six of 37 ) in the osteosynthetic groups. The rates of re-operation for prostheses compared with reconstruction nails were almost double, $8 \%$ vs $14 \%$ (three of 22). In the femoral head and neck, endoprostheses were almost exclusively used and comparison between endoprosthetic and osteosynthetic reconstruction was not possible. For 40 intertrochanteric fractures, the prosthetic rate of re-operation was $10.7 \%$ (three of 28 ) compared with $25 \%$ (three of 12) for osteosynthetic devices. For 51 subtrochanteric fractures, it was $7.4 \%$ (two of 27 ) compared with $12.5 \%$ (three of 24) for osteosynthetic reconstructions. Between 1992 and 2003, three reconstruction nails used for subtrochanteric lesions failed at seven, 11 and 27 months after operation because of nonunion. We conclude that reconstruction nails should be used with caution in patients with a good prognosis.

A complete fracture was seen in $86 \%$ of the lesions and only $14 \%$ of the patients were treated prophylactically. Some authors recommend more frequent use of prophylactic surgery. ${ }^{11}$ With wide indications, more patients in the early stages of disease will be operated on when the potential healing of the fracture is better, leading to fewer failures of osteosynthesis. ${ }^{12}$ However, this may result in unnecessary operations since pathological fractures may be prevented by radiotherapy, bisphosphonates and other oncological treatments. Two large trials of radiotherapy for bone metastases had a rate of fracture of only $5 \%$ and $10 \%$, respectively. ${ }^{13,14}$

It has been reported that the risk of pathological fracture in prostate cancer is low since the lesions are predominantly blastic. ${ }^{15,16}$ In our series, prostate cancer was the second most common primary tumour and all 37 cases were associated with a complete pathological fracture, possibly because the sclerotic bone became increasingly brittle and prone to fracture.

This retrospective study with a mixture of tumours and methods of surgical treatment prevents definite conclusions being made on the ideal operative management of pathological fracture of the proximal femur. However, the study was based on a large database of prospectively collected material without loss to follow-up. The results support our previous opinion that endoprosthetic replacement may be a safer method than osteosynthesis in the proximal femur. Endoprostheses are not dependent on healing of the fracture, which is often poor in patients with cancer because of systemic and local factors. Endoprostheses replace bone whereas osteosynthetic implants are at best load-sharing and will ultimately fail if the bone does not heal. However, dislocations after endoprosthetic replacement are of major concern and efforts to reduce this complication are necessary.

\section{Supplementary Material}

$\because$ A further opinion by Mr Robert Grimer is available with the electronic version of this article on our website at www.jbjs.org.uk

No benefits in any form have been received or will be received from a commercial party related directly or indirectly to the subject of this article. 


\section{References}

1. Wedin R, Bauer HC, Wersäll P. Failures after operation for skeletal metastatic lesions of long bones. Clin Orthop 1999;358:128-39.

2. Johansson T, Jacobsson SA, Ivarsson I, Knutsson A, Wahlström 0. Internal fixation versus total hip arthroplasty in the treatment of displaced femoral neck fractures: a prospective randomized study of 100 hips. Acta Orthop Scand 2000;71: $597-602$

3. Ravikumar KJ, Marsh G. Internal fixation versus hemiarthroplasty versus total hip arthroplasty for displaced subcapital fractures of femur: 13 year results of a prospective randomized study. Injury 2000;31:793-7.

4. Lewallen DG, Berry DJ. Femoral fractures associated with hip arthroplasty. In: Morrey BF, ed. Reconstructive surgery of the joints. New York: Churchill-Livingstone, 1996:1273-88.

5. Patterson BM, Healey JH, Cornell CN, Sharrock NE. Cardiac arrest during hip arthroplasty with a cemented long-stem component: a report of seven cases. J Bone Joint Surg [Am] 1991;73-A:271-7.

6. Kerr PS, Jackson M, Atkins RM. Cardiac arrest during intramedullary nailing for femoral metastases. J Bone Joint Surg [Br] 1993;75-B:972-3.

7. Persson EV, Bauer HCF. Sudden hypotension and profuse bleeding during intramedullary nailing of the femur in cancer patients. Acta Orthop Scand 1994;65: $564-7$.
8. Sim FH, Daugherty TW, Ivins JC. The adjunctive use of methylmethacrylate in fixation of pathological fractures. J Bone Joint Surg [Am] 1974;56-A:40-8.

9. Harrington KD, Sim FU, Enis J0, et al. Methylmethacrylate as an adjunct in internal fixation of pathological fractures: experience with three hundred and seventy-five cases. J Bone Joint Surg [Am] 1976;58-A:1047-55.

10. Ryan JR, Begeman PC. The effects of filling experimental large cortical defects with methylmethacrylate. Clin Orthop 1984;185:306-10.

11. Ward WG, Holsenbeck S, Dorey FJ, Spang J, Howe D. Metastatic disease of the femur: surgical treatment. Clin Orthop 2003;415(Suppl):230-44.

12. Gainor BJ, Buchert P. Fracture healing in metastatic bone disease. Clin Orthop 1983;p178:297-302.

13. Steenland $\mathbf{E}$, Leer $\mathbf{J}$, van Houwelingen $\mathbf{H}$, et al. The effect of a single fraction compared to multiple fractions on painful bone metastases: a global analyses of the Dutch Bone Metastasis Study. Radiother Oncol 1999;52:101-9.

14. No authors listed. 8 Gy single fraction radiotherapy for the treatment of metastatic skeletal pain: randomised comparison with a multifraction schedule over 12 months of patient follow-up. Radiother Oncol 1999;52:111-21.

15. Zickel RE, Mouradian WH. Intramedullary fixation of pathological fractures and lesions of the subtrochanteric region of the femur. J Bone Joint Surg [Am]1976;58-A:1061-6.

16. Mirels H. Metastatic disease in long bones: a proposed scoring system for diagnosis impending pathologic fractures. Clin Orthop 1989;249:256-64. 